\title{
An EEG based Channel Optimized Classification Approach for Autism Spectrum Disorder
}

\author{
Dilantha Haputhanthri \\ Department of Computer \\ Science and Engineering \\ University of Moratuwa \\ Sri Lanka \\ dilantha.15@cse.mrt.ac.lk
}

\author{
Gunavaran Brihadiswaran \\ Department of Computer \\ Science and Engineering, \\ University of Moratuwa \\ Sri Lanka \\ gunavaran.15@cse.mrt.ac.lk
}

\author{
Sahan Gunathilaka \\ Department of Computer \\ Science and Engineering, \\ University of Moratuwa \\ Sri Lanka \\ sahanruwanga.15@cse.mrt.a \\ c.lk
}

\author{
Dulani Meedeniya \\ Department of Computer \\ Science and Engineering, \\ University of Moratuwa \\ Sri Lanka \\ dulanim@cse.mrt.ac.lk
}

\author{
Yasith Jayawardena \\ Department of Computer Science, \\ Old Dominion University, \\ Virginia, USA \\ yasith@cs.odu.edu
}

\author{
Sampath Jayarathna \\ Department of Computer Science, \\ Old Dominion University, \\ Virginia, USA \\ sampath@cs.odu.edu
}

\author{
Mark Jaime \\ Division of Science, \\ Indiana University-Purdue University, \\ Indiana, USA \\ mjaime@iupuc.edu
}

\begin{abstract}
Autism Spectrum Disorder (ASD) is a neurodevelopmental condition which affects a person's cognition and behaviour. It is a lifelong condition which cannot be cured completely using any intervention to date. However, early diagnosis and follow-up treatments have a major impact on autistic people. Unfortunately, the current diagnostic practices, which are subjective and behaviour dependent, delay the diagnosis at an early age and makes it harder to distinguish autism from other developmental disorders. Several works of literature explore the possible behaviour-independent measures to diagnose ASD. Abnormalities in EEG can be used as reliable biomarkers to diagnose ASD. This work presents a low-cost and straightforward diagnostic approach to classify ASD based on EEG signal processing and learning models. Possibilities to use a minimum number of EEG channels have been explored. Statistical features are extracted from noise filtered EEG data before and after Discrete Wavelet Transform. Relevant features and EEG channels were selected using correlation-based feature selection. Several learning models and feature vectors have been studied and possibilities to use the minimum number of EEG channels have also been explored. Using Random Forest and Correlation-based Feature Selection, an accuracy level of $\mathbf{9 3 \%}$ was obtained.
\end{abstract}

Keywords-Autism Spectrum Disorder, EEG signal processing, Discrete Wavelet Transform, classification algorithms

\section{INTRODUCTION}

Autism Spectrum Disorder (ASD) is a complex developmental condition characterized by deficits in social interaction, communication, and restricted and repetitive behaviour. Autism is called a spectrum disorder since there are several types, and the severity of symptoms vary across individuals. It includes three diagnoses: autistic disorder, Asperger's syndrome and pervasive developmental disorder not otherwise specified (PDD-NOS). Autistic people would have communication and behavioural issues, including lack of eye contact and facial expressions. Some children also face intellectual delays. Severe forms of autism might even lead to self-injurious behaviours, seizures, and mental illnesses.

According to a recent CDC report, 1 in 59 children suffers from ASD [1]. Studies have shown that the prevalence of autism is increasing over the years [2]. An early diagnosis would assist early interventions, which in turn might increase the child's response rate to treatments. Social skills training at an early age has a powerful impact on reversing the symptoms, thus facilitating the autistic people to lead a healthy life. The exact cause for ASD has not been found so far, and no biological tests exist to diagnose ASD. Besides, the current diagnostic practices are based solely on behavioural patterns. Because of that diagnosis before the age of three is difficult as the defining behaviours do not appear at early ages and there are not any simple measurements which could be implemented routinely during the well-baby check-ups. Milder forms of ASD are even harder to diagnose at an early age because the neurodevelopmental symptoms are common to several diagnoses. The fact that with time, etiology and development course become more diverse makes the early diagnosis even more challenging. These drawbacks also lead to misdiagnosis [3].

Several studies have been carried out to find potential, behaviour-independent biomarkers for autism. Many studies have illustrated the correlation between ASD, EEG signals, joint attention, and eye movement [4][5][6]. These diagnostic approaches are expected to be less expensive and easy to implement so that they can be incorporated into the routine well-baby check-ups. Recently, signal processing of EEG data, image processing of fMRI data together with feature extraction and learning models have been researched as a potential approach for classifying ASD.

In this paper, we present an efficient and low-cost approach based on EEG signal processing, statistical feature extraction, and learning models, that can be used to diagnose ASD. The primary goal of this study is to construct learning models based on features obtained from noise filtered, discrete, the optimum number of EEG channels. This paper explores the minimum number of channels required to train the learning models so that the time and cost spent on obtaining EEG data can be reduced. The proposed approach has been implemented in a prototype named ASDGenus. We have managed to achieve $93.33 \%$ accuracy using only five channels, unlike the other existing works.

The paper is structured as follows: Section II explores the existing literature, Section III describes the methodology

This is the author's manuscript of the work published in final edited form as: 
including the subjects involved, the dataset and the system model, Section IV discusses the results, and Section V concludes the paper.

\section{BACKGROUND}

Abnormalities in brain signals used by brain cells to communicate with each other can be used to characterize neurodevelopmental disorders in the early ages before the appearance of behavioural symptoms. Several studies have been carried out previously to find potential behaviour independent biomarkers for autism. These studies show that electroencephalogram (EEG) signals have strong correlations with ASD and can be used as a reliable biomarker [7]. EEG captures the electrical impulses used by the brain cells to communicate with each other through electrodes which are attached to the scalp. The time series obtained from EEG facilitates the observation and analysis of the abnormalities in the underlying neural network.

Pre-processing and feature extraction are necessary steps when using EEG data to train learning models. EEG data contain Ocular (eye blink) and Myogenic artefacts as noise. Several techniques, including Independent Component Analysis (ICA), Statistical Analysis, and Wavelet-Based Analysis, are used for noise removal [8][9]. For feature extraction, techniques such as Principal Component Analysis (PCA) [10] and Discrete Wavelet Transform (DWT) [11] are widely used. The wavelet transform is a non-stationary, timescale analysis technique which can be used to separate the given signal into frequency elements in various time scales. DWT is the implementation of the wavelet transform over discrete sets of wavelet scales. Table I summarizes the techniques used in the previous studies.

Bosl et al. followed a data-driven approach for the early diagnosis of autism with the help of EEG data [12]. Data were obtained from 89 low-risk controls (LRC) and 99 high-risk for autism (HRA), a total of 188 participants. Participants in low-risk control had at least one typically developing sibling and no first degree relative with autism, while participants in high-risk control had a sibling with ASD. They used nine non-linear features including sample entropy, detrended fluctuation analysis, entropy derived from recurrence plot, max line length, mean line length, recurrence rate, determinism, laminarity, and trapping time. Using Support Vector Machine (SVM), they were able to distinguish ASD from LRC with $100 \%$ accuracy and sensitivity. The predicted severity scores had a strong correlation with the actual severity scores as well. However, sensitivity and accuracy for classifying HRA infants were comparatively low.

Abdulhay et al. studied the inter-channel stability of EEG signals and frequency 3D mapping to investigate the potential for detecting irregularities in EEG signals and connectivity with Autism Spectrum Disorder [13]. EEG data were collected from 20 participants (between 4 to 13 years of age), 10 autistic children and 10 neuro-typical children, using a 64channel cap according to the International 10-20 system. Selected EEG signals were filtered using a band-pass filter to ensure the range of $0.3-40 \mathrm{~Hz}$ and noises were removed using ICA method via LA-106 ASA ERP software and by a neurologist. Then, each EEG channel was decomposed by the Empirical Mode Decomposition (EMD) method to extract the Intrinsic Mode Function (IMF). Analytic IMF, Local pointby-point pulsation, Stability loop, and Frequency 3D mapping were used as features in their study. The study found that the inter-channel stability of pulsation plot and the distribution of frequency content throughout the scalp were promising indicators for autism.

Another new complex system based on Artificial Neural Networks (ANNs) was developed using Multi-Scale Ranked Organizing Map coupled with Implicit Function as Squashing Time (MS-ROM/I-FAST) algorithm. One key feature of this system is that it was able to extract features from EEG data without any preliminary pre-processing

TABLE I. Summary OF DATA PRE-PROCESSING AND ClassificATION TECHNIQUES

\begin{tabular}{|c|c|c|c|c|c|c|c|c|c|c|c|c|c|c|c|c|c|}
\hline \multirow[b]{2}{*}{ Related Study Description } & \multirow[b]{2}{*}{$\begin{array}{c}\text { Dataset } \\
\text { Type }\end{array}$} & \multicolumn{9}{|c|}{ Data pre-processing techniques } & \multicolumn{7}{|c|}{ Classification techniques } \\
\hline & & 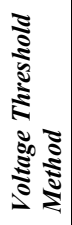 & 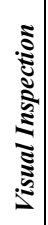 & 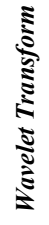 & క্র & $\frac{5}{5}$ & 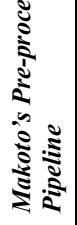 & $\begin{array}{l}3 \\
\vdots \\
\vdots \\
\vdots \\
\vdots \\
0 \\
0\end{array}$ & 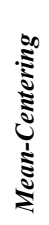 & 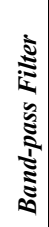 & $\stackrel{5}{5}$ & 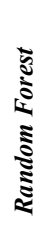 & $\vdots$ & $\gtreqless$ & 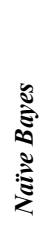 & 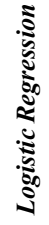 & 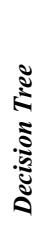 \\
\hline $\begin{array}{l}\text { W. Bosl, Tierney, Tager-Flusberg, \& } \\
\text { Nelson, 2011 [7] }\end{array}$ & EEG & & & & & & & & & $\mathrm{X}$ & $X$ & & $\mathrm{X}$ & & $X$ & & \\
\hline Bosl, Tager-Flusberg, \& Nelson, 2018 [12] & EEG & & & $\mathrm{X}$ & & & & & & $\mathrm{X}$ & $\mathrm{X}$ & $\mathrm{X}$ & $\mathrm{X}$ & & & & \\
\hline $\begin{array}{l}\text { Abdulhay, Alafeef, Hadoush, Alomari, \& } \\
\text { Bashayreh, } 2017 \text { [13] }\end{array}$ & EEG & & & & $\mathrm{X}$ & & & & & & & & & & & & \\
\hline Thapaliya, Jayarathna, \& Jaime, 2018 [14] & $\begin{array}{l}\text { EEG, Eye } \\
\text { movement }\end{array}$ & & $X$ & & & & $\mathrm{X}$ & & & & $\mathrm{X}$ & & & $X$ & $X$ & $X$ & \\
\hline Sahroni, Igasaki, \& Murayama, 2015 [15] & EEG & & & & & & & & & $\mathrm{X}$ & & & & & & & \\
\hline Grossi, Olivieri, \& Buscema, 2017 [16] & EEG & & & & & $\mathrm{X}$ & & & & & & $\mathrm{X}$ & $\mathrm{X}$ & $\mathrm{X}$ & $\mathrm{X}$ & $\mathrm{X}$ & \\
\hline Jiang \& Zhao, 2017 [17] & $\begin{array}{l}\text { Eye } \\
\text { movement }\end{array}$ & & & & & & & $X$ & & & & & & $\mathrm{X}$ & & & \\
\hline Fan et al., 2018 [18] & EEG & & & & & & & & $\mathrm{X}$ & $\mathrm{X}$ & $\mathrm{X}$ & $\mathrm{X}$ & $\mathrm{X}$ & $\mathrm{X}$ & $\mathrm{X}$ & & $\mathrm{X}$ \\
\hline Harun et al., 2018 [19] & EEG & & & & & & & & & $\mathrm{X}$ & $\mathrm{X}$ & & & $\mathrm{X}$ & & & \\
\hline Cheong, Sudirman, \& Hussin, 2015 [20] & EEG & $\mathrm{X}$ & & $\mathrm{X}$ & & & & & & $\mathrm{X}$ & & & & $\mathrm{X}$ & & & \\
\hline
\end{tabular}


I-FAST algorithm consists of three phases: squashing phase, noise elimination phase, and a classification phase. Similarly, in MS-ROM, there are three steps: sampling, projection, and ranking. Using 60 seconds long EEG data obtained from 25 participants, 15 ASD and 10 typically developing, and machine learning models they managed to achieve $100 \%$ accuracy using training-testing protocol and $84 \%-92.8 \%$ accuracy using leave one out protocol.

Even though several works have studied EEG and eye movement separately to find correlations to autism, Thapaliya et al. demonstrated the possibility for combining both the EEG and eye movement [14]. Using the data obtained from 52 participants, $24 \mathrm{ASD}$ and 28 control, they studied the identification of ASD and presented the comparison of the performances of different machine learning algorithms. Using EEGLab and applying Makoto's Pre-processing Pipeline and visual inspection, the EEG data were pre-processed. Statistical (mean, standard deviation, and combined mean and standard deviation) and entropy values were used as features. They compared SVM, Logistic Regression, DNN, and Gaussian Naïve Bayes, and the best results were obtained when using Logistic Regression with a combination of EEG and eye tracking data. The achieved accuracy was $100 \%$.

Even though the existing studies have produced significant results, most of them have used either 64 or 128 channel EEG data. The generated systems remain a complete black box without any medical interpretation. By reducing the number of channels required for identifying ASD, real-world practical implementation can be further improved by simplifying the procedure and increasing the affordability. In addition, by reducing the number of channels as well as the number of features the interpretability of the system, one of the crucial aspects in the medical field, can be increased.

\section{Methodology}

\section{A. Study Population and EEG Data Processing}

The dataset used was a subset of data from the previous work [21]. The study includes 15 participants, 9 males, and 6 females, between 5 and 17 years of age. 10 of them had a prior ASD diagnosis, and the remaining 5 did not. In order to obtain the optimum results using data from 15 subjects and to increase the reliability, we have used k-fold cross-validation approach. EEG data were collected during the ADOS-2 assessment. A 32 channel LiveAmp wireless EEG system with active electrodes was used, and the signals were sampled at $250 \mathrm{~Hz}$. Fig. 1 and Fig. 2 shows the electrode locations [22] and the raw EEG signals of the 32 channels, respectively.

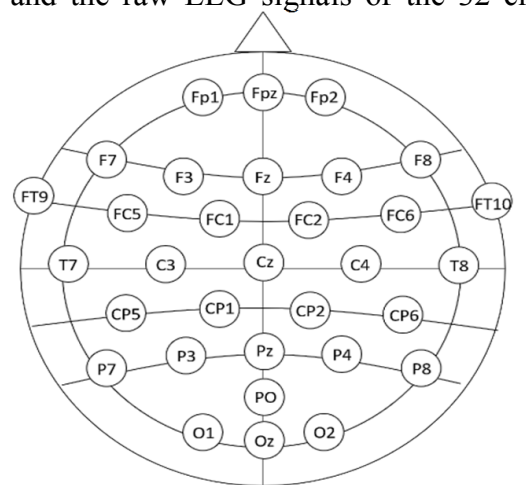

Fig. 1. 32 electrode locations of the EEG channels

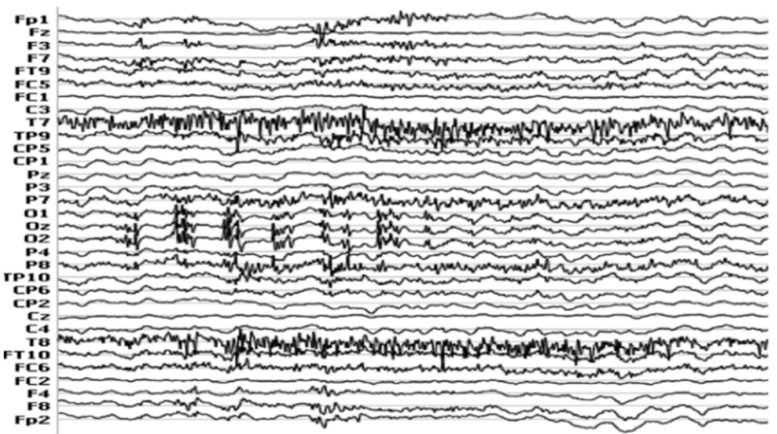

Fig. 2. EEG signals of 32 channels

The channels were recorded using $\mathrm{FCz}$ electrode as reference. The data were gathered for 15 minutes on average. Autism Diagnostic Observation Schedule, second edition (ADOS-2) score, and Autism Spectrum Quotient (AQ) values were calculated. ADOS modules 3 and 4 were used.

The noise from eye-blink was filtered using a simple customized algorithm. The idea is to remove the excessive signal patterns when they exceed a threshold level. The discrete signal was traversed, and the outliers were substituted with the cumulative mean. When traversing each data point, the cumulative mean was updated. In Fig. 3, the original signal is shown by blue colour lines and the noise removed signal is indicated by orange colour lines. In this process, eye-blink noise is filtered based on (1). Here, we have defined a threshold $\mathrm{T}_{0}$ based on visual inspection of the EEG signal in order to identify the abnormal values. If the absolute value of the amplitude is above $T_{0}$, the amplitude is substituted with the cumulative mean, which is the average value of the previously traversed data points.

$$
x[n]= \begin{cases}x[n] ; & \text { if }|x[n]| \leq T_{0} \\ \frac{1}{n-1} \sum_{k=1}^{n-1} x[k] ; & \text { if }|x[n]|>T_{0}\end{cases}
$$

\section{B. ASD Classification Model}

Since the early intervention of ASD and therapies could increase the response rate of autistic people, the diagnostic approach should be simple, low-cost, and easy to implement. The methodology is a three-phase process: pre-processing, feature extraction, and classification. The system model is shown in Fig. 4. In the pre-processing stage, the noise from eye-blink is filtered.

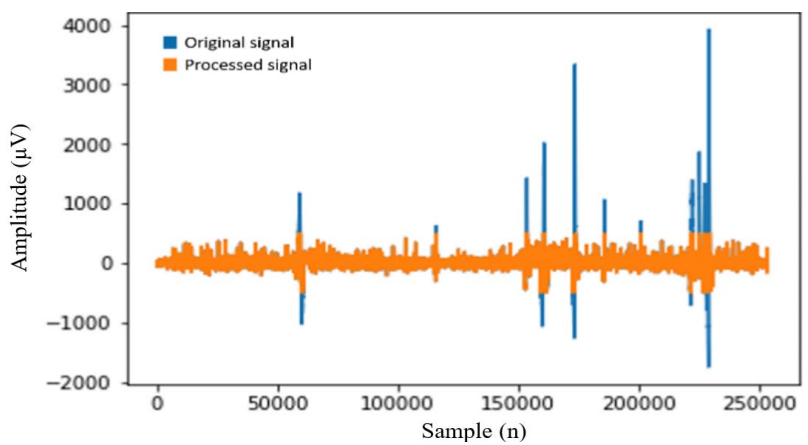

Fig. 3. Original and noise removed discrete EEG signals 


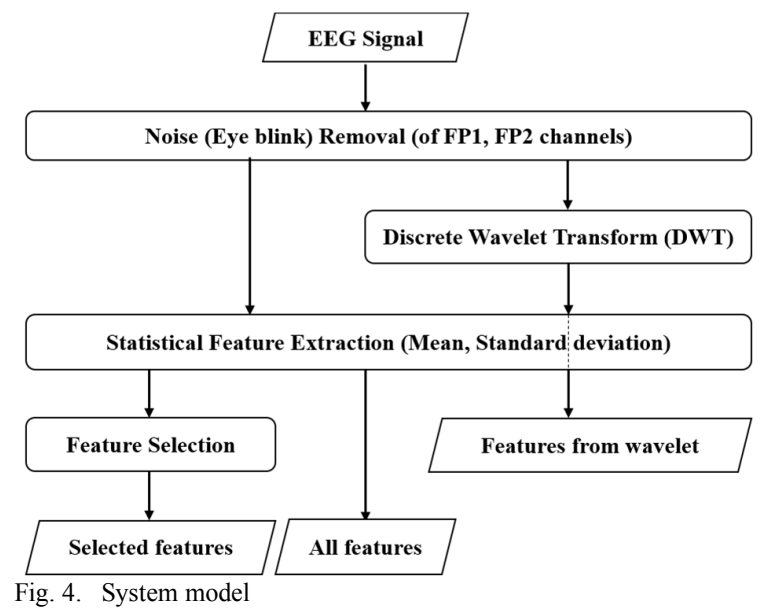

The feature extraction phase comprises of two components. One component extracts statistical features from the noise-removed EEG signals and the other extracts the statistical features after DWT. We calculated the mean and standard deviation of each channel and the feature vectors consisting of these results.

Studies have shown that the beta frequency band has a considerable influence on classifying ASD [15]. Using DWT, we were able to divide the discrete signal into frequency bands. The decomposition of the DWT is computed by filtering the discrete signal. This filtering uses a low pass filter to obtain the approximation coefficient (CA) and a high pass filter to obtain the detailed coefficient (CD) [20]. The frequency range of the beta band is from $16 \mathrm{~Hz}$ to $32 \mathrm{~Hz}$. The frequency of the original EEG signal was $250 \mathrm{~Hz}$. Thus, DWT had to be performed up to four levels. The procedure is shown in Fig. 5. Then, the statistical features of the beta frequency band were extracted.

The extracted statistical features are the mean and standard deviation of the channels. There are 32 channels in the EEG data and two features (mean and standard deviation) for each channel, thus 64 feature vectors in total. We tested four different models using six different feature sets: FS1, FS2, FS3 - set of all 64 extracted features before and after DWT and their combination, FS4 - features selected based on Correlation-based Feature Selection (CFS), FS5 - means and standard deviations of 5 channels included in the output of CFS algorithm and FS6 - means and standard deviations of channels used in the International 10-20 System (Fp1, Fp2, F7, F3, Fz, F4, F8, T3, C3, Cz, C4, T4, T5, P3, Pz, P4, T6, $\mathrm{O} 1, \mathrm{O} 2$ and Common Ground as shown in Fig. 1).

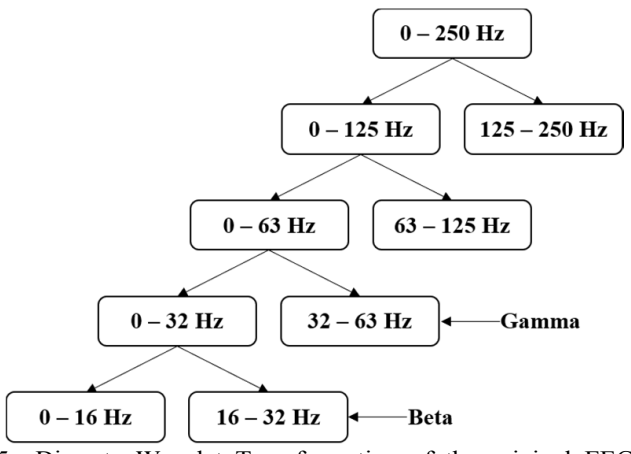

Fig. 5. Discrete Wavelet Transformation of the original EEG signal to separate the beta frequency band
CFS algorithm selects useful features based on a strong correlation to the classification. International $10-20$ system is an internationally recognized method of electrode placement on the scalp. In the available dataset, the channels T3, T4, T5, and $\mathrm{T} 6$ were not available. Thus, we carried out the tests using the remaining 15 channels. Four learning models (Logistic Regression, Support Vector Machine (SVM), Naïve Bayes and Random Forest) were trained for the classification and validated using cross-validation method. Cross-validation was used for a smaller number of subjects.

\section{Implementation Details}

The DWT was implemented using the PyWavelets library based on the mathematical model shown in Fig. 6. The approximation coefficient and detailed coefficient mentioned in section B were calculated using band-pass filters $\mathrm{g}(\mathrm{n})$ and $h(n)$. Here, $x[n]$ indicates the input signal, while $y[n]$ indicates the output signal of each bandpass filter.

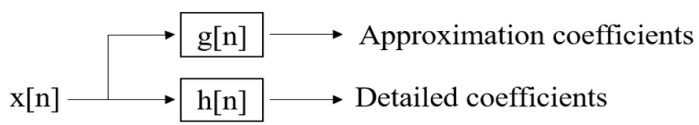

Fig. 6. Wavelet decomposition of EEG signal

The approximation coefficient is obtained by the low-pass filter $\mathrm{g}(\mathrm{n})$ as defined in (2)

$$
g[n]: \quad y[n]=\alpha \cdot x[n]+(1-\alpha) \cdot y[n-1]
$$

where smoothing factor $\alpha=\frac{\Delta T}{R C+\Delta T}$

The detailed coefficient is obtained by the high-pass filter $\mathrm{h}(\mathrm{n})$ as defined in (3).

$$
\begin{aligned}
& h[n]: \quad y[n]=\alpha \cdot y[n-1]+\alpha(x[n]-x[n-1]) \\
& \text { where smoothing factor } \alpha=\frac{R C}{R C+\Delta T} \\
& \text { equivalent time constant } \mathrm{RC}=\frac{1}{2 \pi f_{c}}
\end{aligned}
$$

where $f_{\mathrm{c}}$ is the cut off frequency, and $\Delta T$ is the sampling period. We have developed a customized algorithm for eyeblink removal and the pseudocode is shown in Algorithm 1.

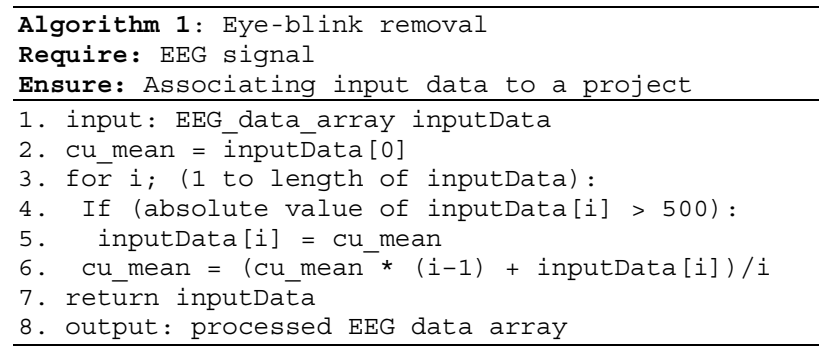

The input to the algorithm is an EEG data array for a channel, which contains the amplitudes of the EEG signal. Then the mean of the channel is initialized as the first element of the EEG data array, assuming that the first value is a valid one (through visual inspection it was confirmed that the signals do not start with abnormal values). Then the absolute value of each amplitude value is checked, if the value is greater than the threshold of 500 microvolts as illustrated in (1), that value is replaced by the channel's cumulative mean. During each iteration, the cumulative mean of the channel is updated. Finally, the updated signal data array is returned. 


\section{EVALUATION}

This section presents the obtained accuracies of the ASDGenus prototype and their interpretations, as given in Table II. For the feature sets FS1, FS2, and FS3 the accuracy of logistic regression and SVM are comparatively lower than Naïve Bayes and random forest. They cannot be used as reliable classifiers using these sets of features. Among the four, the Naive Bayes model shows comparatively high accuracy close to $75 \%$.

In the feature set FS4, which is obtained using the CFS algorithm, the selected features are means of FT9, P3 and Oz channels, and standard deviations of TP9 and FC2 channels (from this point onwards these five features will be called
Selected Features). Similar to the previous case, SVM does not give good accuracy. However, logistic regression and random forest exhibit high accuracies above $87 \%$. The feature set FS5 includes the means, and standard deviations of all the five channels (FT9, P3, Oz, TP9 and FC2) included in the feature set selected by the CFS algorithm (FS4).

From this point onwards these five channels will be called the Selected Channels. Again, SVM does not show a high accuracy level. As shown in Table II, logistic regression produced the highest accuracy level of $87 \%$. For the feature set FS6, compared to the results of features set FS5, the obtained accuracy levels are relatively low. Naive Bayes has a maximum accuracy of $74 \%$.

TABLE II. SUMMARY OF TEST RESULTS OBTAINED USING DIFFERENT FEATURE SETS AND LEARNING ALGORITHMS

\begin{tabular}{|c|c|c|c|c|c|c|}
\hline & $\begin{array}{c}\text { All Statistical } \\
\text { Features (FS1) } \\
\end{array}$ & $\begin{array}{c}\text { Statistical Features } \\
\text { After DWT (FS2) }\end{array}$ & $\begin{array}{c}\text { Combined Feature } \\
\text { Set (FS3) } \\
\end{array}$ & $\begin{array}{c}\text { Selected Feature } \\
\text { Set (FS4) }\end{array}$ & $\begin{array}{c}\text { Selected } \\
\text { Channels (FS5) } \\
\end{array}$ & $\begin{array}{l}\text { International 10- } \\
20 \text { System (FS6) }\end{array}$ \\
\hline Logistic Regression & $46.66 \%$ & $53.33 \%$ & $53.33 \%$ & $86.66 \%$ & $86.66 \%$ & $60.00 \%$ \\
\hline SVM & $53.33 \%$ & $60 \%$ & $53.33 \%$ & $46.66 \%$ & $53.33 \%$ & $53.33 \%$ \\
\hline Naive Bayes & $73.33 \%$ & $73.33 \%$ & $73.33 \%$ & $73.33 \%$ & $73.33 \%$ & $73.33 \%$ \\
\hline Random Forest & $66.66 \%$ & $66.66 \%$ & $66.66 \%$ & $93.33 \%$ & $73.33 \%$ & $66.66 \%$ \\
\hline
\end{tabular}

Fig. 7 shows the summarized test results graphically. The highest accuracy of 93\% is obtained when Random Forest and feature set selected through CFS are coupled together. Logistic Regression also yields an accuracy of $87 \%$ when used with selected feature set or with all the features of the selected channels. Compared to other learning models, the results produced by SVM are relatively low. It never surpassed the $60 \%$ accuracy mark. The accuracy levels produced by the Naïve Bayes model are consistent at $74 \%$ regardless of the feature set. Furthermore, the feature set selected using CFS provides comparatively higher accuracy levels except for SVM.

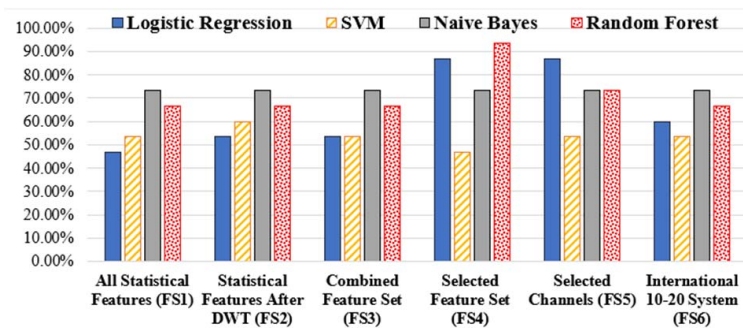

Fig. 7. Graphical representation of the comparison of all the test results

Initially, we started testing the learning models with all the 64 feature vectors (FS1). Then we removed 13 channels and tried using the channels in the International 10-20 System (FS6). We reduced the feature vectors by 24. After this modification, the accuracy of the logistic regression improved from $47 \%$ to $60 \%$. However, the others remained unchanged. Then we used the CFS and selected 5 channels included in the results (FS5) (10 feature vectors in total). It improved the accuracy of Logistic Regression considerably from $60 \%$ to $87 \%$.

The accuracy of the random forest algorithm also improved from $67 \%$ to $74 \%$. Finally, only the 5 features selected by the CFS were used (FS4). Further, it has improved the accuracy of the random forest to $93 \%$. On the contrary, the accuracy of SVM dropped to $47 \%$. Although we have tried different sets of features extracted after DWT (FS2), it has improved the accuracy of logistic regression from $47 \%$ to $54 \%$. Moreover, the combination of all the extracted features before and after DWT (FS3) does not produce better results. The accuracy level drops for SVM and remains unchanged for other learning models. As shown in Table III, we managed to obtain a comparable accuracy of $93 \%$ using only 5 channels, unlike the other studies.

TABLE III. COMPARISON OF BEST ACCURACIES

\begin{tabular}{|l|c|c|}
\hline \multicolumn{1}{|c|}{ Related Study } & $\begin{array}{c}\text { No. of } \\
\text { Channels }\end{array}$ & $\begin{array}{c}\text { Best } \\
\text { Accuracy }\end{array}$ \\
\hline $\begin{array}{l}\text { ASD classification using EEG and eye } \\
\text { movement [14] }\end{array}$ & 128 & $100 \%$ \\
\hline A data-driven approach to classify ASD [12] & 19 & $100 \%$ \\
\hline $\begin{array}{l}\text { Classifying ASD using MS-ROM/I-FAST } \\
\text { algorithm [16] }\end{array}$ & 19 & $100 \%$ \\
\hline $\begin{array}{l}\text { EEG as a biomarker for classifying autistic } \\
\text { children [7] }\end{array}$ & 64 & $90 \%$ \\
\hline ASDGenus & 5 & $93.33 \%$ \\
\hline
\end{tabular}

\section{CONCLUSION}

ASD is a developmental disorder that affects social interaction, communication, and behaviours of a person and may impact on neurological comorbidities. Even though a specific test to identify the severity of ASD outright does not exist, different neurological tests using EEG and eye movement data are being studied for the identification purpose. This paper is focused on an automated approach for early intervention of ASD classification using a minimum number of EEG channels to simplify the process and to increase the affordability, thus enabling better individual treatments. We have mainly considered the feature selection and extraction of EEG signals with the aim of classifying data using a minimum number of channels and machine learning approaches for better decision making. The proposed approach was implemented via the prototype ASDGenus.

We have developed an EEG signal processing algorithm to filter eye blink noise. The feature extraction is done using statistical approaches and discrete wavelet transform based approach. In this paper, we have compared four different earning models, including logistic regression, SVM, Naïve Bayes, and random forest. Moreover, we have considered six different feature sets FS1-FS6. 
According to the obtained results, the random forest model coupled with CFS produce the best results with an accuracy of 93\%. Logistic regression, coupled with feature sets FS4 and FS5, also produce promising results with an accuracy of $87 \%$. The results obtained from SVM were not satisfactory. The initial tests were carried out using 32 channels, and then in the next stages, the number of channels was reduced to 19 and finally further reduced to 5 . We intend to make this objective approach easy to implement during the routine well-baby checkups by reducing the number of channels that make this procedure simple, and time and cost effective. We intend to combine EEG data and thermal imaging data to improve prediction accuracy as future work. Thus, the proposed approach can be extended using thermal image processing techniques and advanced learning models to provide a betterautomated decision support system for ASD identification.

\section{ACKNOWLEDGEMENT}

This work was supported by Old Dominion University, Norfolk, Virginia. Authors would also like to thank Indiana University-Purdue University, Columbus, Indiana for providing the EEG dataset for the experiments. Also, we acknowledge the support received from the Senate Research Committee Grant SRC/LT/2019/18, University of Moratuwa, Sri Lanka.

\section{REFERENCES}

[1] J. Baio et al., "Prevalence of Autism Spectrum Disorder Among Children Aged 8 Years - Autism and Developmental Disabilities Monitoring Network, 11 Sites, United States, 2014," $M M W R$. Surveillance Summaries, vol. 67, no. 6, pp. 1-23, Apr. 2018.

[2] J. L. Matson and A. M. Kozlowski, "The increasing prevalence of autism spectrum disorders," Research in Autism Spectrum Disorders, vol. 5, no. 1, pp. 418-425, Mar. 2011.

[3] D. S. Mandell, R. F. Ittenbach, S. E. Levy and J. A. Pinto-Martin, "Disparities in Diagnoses Received Prior to a Diagnosis of Autism Spectrum Disorder," Journal of Autism and Developmental Disorders, vol. 37, no. 9, pp. 1795-1802, Oct. 2007.

[4] L. M. Oberman, E. M. Hubbard, J. P. McCleery, E. L. Altschuler, V. S Ramachandran and J. A. Pineda, "EEG evidence for mirror neuron dysfunction in autism spectrum disorders," Cognitive Brain Research, vol. 24, no. 2, pp. 190-198, Jul. 2005.

[5] P. Mundy, "A review of joint attention and social-cognitive brain systems in typical development and autism spectrum disorder," European Journal of Neuroscience, vol. 47, no. 6, pp. 497-514, Sep. 2018.

[6] L. Billeci et al., "An integrated EEG and eye-tracking approach for the study of responding and initiating joint attention in Autism Spectrum Disorders," Scientific Reports, vol. 7, pp. 1-13, Oct. 2017.

[7] W. Bosl, A. Tierney, H. Tager-Flusberg and C. Nelson, "EEG complexity as a biomarker for autism spectrum disorder risk," $B M C$ Medicine, vol. 9, no. 18, p. 1-16, Feb. 2011
[8] C. Q. Lai, H. Ibrahim, M. Z. Abdullah, J. M. Abdullah, S. A. Suandi and A. Azman, "Artifacts and noise removal for electroencephalogram (EEG): A literature review," in IEEE Symposium on Computer Applications \& Industrial Electronics (ISCAIE), 2018, pp. 326-332.

[9] P. N. Jadhav, D. Shanamugan, A. Chourasia, A. R. Ghole, A. Acharyya and G. Naik, "Automated detection and correction of eye blink and muscular artefacts in EEG signal for analysis of Autism Spectrum Disorder," in 36th Annual International Conference of the IEEE Engineering in Medicine and Biology Society, 2014, pp. 1881-1884.

[10] J. Shlens, "A Tutorial on Principal Component Analysis," arXiv Prepr. arXiv1404.1100, Apr. 2014

[11] C. Y. Jung and S. S. Saikiran, "A review on EEG artifacts and its different removal technique," Asia-pacific Journal of Convergent Research Interchange, vol. 2, no. 4, pp. 43-60, Dec. 2016.

[12] W. J. Bosl, H. Tager-Flusberg and C. A. Nelson, "EEG Analytics for Early Detection of Autism Spectrum Disorder: A data-driven approach," Scientific Reports, vol. 8, no. 1, pp. 1-20, May. 2018.

[13] E. Abdulhay, M. Alafeef, H. Hadoush, N. Alomari and M. Bashayreh, "Frequency 3D mapping and inter-channel stability of EEG intrinsic function pulsation: Indicators towards autism spectrum diagnosis," in 10th Jordanian International Electrical and Electronics Engineering Conference (JIEEEC), 2017, pp. 1-6.

[14] S. Thapaliya, S. Jayarathna and M. Jaime, "Evaluating the EEG and Eye Movements for Autism Spectrum Disorder," in IEEE International Conference on Big Data (Big Data), 2018, pp. 2328-2336.

[15] A. Sahroni, T. Igasaki and N. Murayama, "Band powers analysis of spontaneous EEG with uncooperative autism children during short sleep condition," in 8th International Conference on Biomedical Engineering and Informatics (BMEI), 2015, pp. 163-168.

[16] E. Grossi, C. Olivieri and M. Buscema, "Diagnosis of autism through EEG processed by advanced computational algorithms: A pilot study," Comput. Methods Programs Biomed., vol. 142, pp. 73-79, Apr. 2017.

[17] M. Jiang and Q. Zhao, "Learning Visual Attention to Identify People with Autism Spectrum Disorder," in IEEE International Conference on Computer Vision (ICCV), 2017, pp. 3287-3296.

[18] J. Fan et al., "A Step towards EEG-based brain computer interface for autism intervention," in 37th Annual International Conference of the IEEE Engineering in Medicine and Biology Society (EMBC), 2015, pp. 3767-3770.

[19] N. Harun, N. Hamzah, N. Zaini, M. Sani, H. Norhazman, and I. Yassin, "EEG Classification Analysis for Diagnosing Autism Spectrum Disorder based on Emotions," Journal of Telecommunication, Electronic and Computer Engineering, vol. 10, no. 1-2, pp. 87-93, 2018.

[20] L. C. Cheong, R. Sudirman, and S. S. Hussin, "Feature extraction of EEG signal using wavelet transform for autism classification," ARPN Journal of Engineering and Applied Sciences, vol. 10, no. 19, pp. 85338540, Oct. 2015.

[21] S. Jayarathna, Y. Jayawardana, M. Jaime, and S. Thapaliya, "Electroencephalogram (EEG) for Delineating Objective Measure of Autism Spectrum Disorder," in Computational Models for Biomedical Reasoning and Problem Solving, IGI Global, 2019, pp. 34-65.

[22] E. Niedermeyer and F. H. L. da Silva, Electroencephalography: basic principles, clinical applications, and related fields, Lippincott Williams \& Wilkins, 2005. 